\title{
Match-to-match variations in external load measures during congested weeks in professional male soccer players
}

\author{
Rui Silva1 , Halil Ibrahim Ceylan², Georgian Badicu ${ }^{3, *}$, Hadi Nobari $^{4,5,6}$, \\ Sílvio Afonso Carvalho ${ }^{7}$, Tiago Sant'Ana ${ }^{1}$, Bruno Mendes ${ }^{8}$, Yung-Sheng Chen ${ }^{9}$, \\ Filipe Manuel Clemente ${ }^{1,10}$
}

\footnotetext{
${ }^{1}$ Escola Superior Desporto e Lazer, Instituto Politécnico de Viana do Castelo, Rua Escola Industrial e Comercial de Nun'Álvares, 4900-347 Viana do Castelo, Portugal

${ }^{2}$ Physical Education and Sports Teaching Department, Kazim Karabekir Faculty of Education, Ataturk University, 25030 Erzurum, Turkey

${ }^{3}$ Department of Physical Education and Special Motricity, University Transilvania of Brasov, 500068 Brasov, Romania

${ }^{4}$ Department of Physical Education and Sports, University of Granada, 18010 Granada, Spain

${ }^{5}$ Department of Exercise Physiology, Faculty of Sport Sciences, University of Isfahan, 81746-7344 Isfahan, Iran

${ }^{6} \mathrm{HEME}$ Research Group, Faculty of Sport Sciences, University of Extremadura, 10003 Cáceres, Spain

${ }^{7}$ Associação de Futebol de Bragança, 5300-379 Bragança, Portugal

${ }^{8}$ Faculty of Human Kinetics, University of Lisboa, 1649-004 Lisboa, Portugal

${ }^{9}$ Department of Exercise and Health Sciences, University of Taipei, 11153 Taipei, Taiwan

${ }^{10}$ Instituto de Telecomunicações, Delegação da Covilhã, 1049-001 Lisboa, Portugal
}

*Correspondence: georgian.badicu@unitbv.ro (Georgian Badicu)

\begin{abstract}
Objectives: This study aimed to analyze within-week and within-match external load variations in male soccer players over three consecutive matches during a congested week.

Methods: The study cohort included nineteen elite professional male players (age: $26.5 \pm 4.3$ years) from a European First League team. Players were monitored daily over a full season using measurements collected by global positioning systems (GPSs). GPS-derived measures of total distance (TD), high-speed running (HSR), high metabolic load (HML), and maximal speed (maxSpeed) were collected during each match.

Results: TD and HML intensity were meaningfully lower during the second half of the season than the first half for all weeks $(p<0.05)$, regardless of the number of matches. Also, the standardized differences for both metrics presented moderate-to-strong effect sizes. Although no significant differences between halves were found for HSR or maxSpeed $(p>0.05)$, these measures presented inconsistently minimum-to-strong effect sizes in some matches in overall weeks.

Conclusion: The findings of this study revealed that TD and HML distances were significantly different between halves for all weeks, regardless of the number of matches. Meanwhile, HSR and maxSpeed measures presented no significant differences across matches overall.
\end{abstract}

\section{Keywords}

External load; Load monitoring; Sports science; Performance; GPS

\section{Introduction}

Soccer represents a complex order versus disorder system that requires the continuous assessment of athletes' work- loads, especially during congested fixtures $[1,2]$. The systematic monitoring of the loads imposed on athletes allows coaches to track the progression and direction of training and match loads [3]. Follow-ups on these types of assessments 
are of paramount importance to make necessary training adjustments, as well as to ensure more individualized recovery strategies considering athletes who play more than one match per week [4, 5].

The process of load monitoring is organized into two main interconnected concepts [6]: (i) external loads (the physical demands imposed on players by coaches) and (ii) internal loads (the psychobiological responses to the imposed demands). Although these two monitoring concepts are related to each other, they are quantified by different means [7]. While internal loads can be measured using objective and subjective measures, external loads can only be quantified through objective devices such as global positioning systems (GPSs), inertial measurement units (IMUs), and accelerometers-the latter are usually embedded in modern GPSs [8]. There are some methodological and practical concerns regarding GPS metrics and the different analyses of external loads. The most common are (i) distances covered at different speed thresholds; (ii) changes in speed, such as accelerations and decelerations; and (iii) actions measured by accelerometers or IMUs, such as high metabolic load and impacts [9].

Weekly soccer schedules have become more congested due to the inclusion of both domestic and international competitions in addition to competitions for each team's national league. Thus, athletes often are given short recovery periods between matches during a training week and, therefore, are at a relatively high risk of suffering an injury [10]. Indeed, exposure to more than two matches during a week is associated with decreased strength and muscle stiffness and increased physiological stress [11-13]. Moreover, matches have residual fatigue effects on players that can last up to $72 \mathrm{~h} \mathrm{[14]}$. Thus, a better understanding is needed of how external demands are handled by players who participate in multiple matches in one week. Although several studies have documented the effects of congested fixtures on soccer players, inconsistencies have arisen regarding the dependencies of some GPS metrics regarding congested schedules, which still to be resolved [15].

Coaches and practitioners now have insights into the match intensity of each GPS-derived metric and can use this information when analyzing the external load demands associated with matches. They can do this by dividing each external load metric by match duration, which yields relativized per-minute values [16]. Overall, studies on this topic have focused on congested fixtures' acute effects on external load demands [15]. However, few studies have analyzed between-match and within-match variations during congested weeks using per-minute external load measures. One previous study used match intensity values instead of volume to analyze between- and within-match variations and found that the overall distances covered per minute varied between matches in congested weeks but only for low-intensity measures [17]. Considering variations within matches during a congested fixture, the same authors [17] revealed that neither low-intensity nor high-intensity measures varied between the two halves of a match.
As has been reported [18, 19], overall external load measures are not affected by a lack of significant differences between congested and non-congested weeks. However, other studies have revealed that this matter may not be so straightforward $[20,21]$. Studies conducted on an elite soccer team from the top "big five" European leagues revealed that both distance- and accelerometry-based volume measures changed during congested fixtures, especially among players who participated in three consecutive matches $[20,21]$. However, the reported dependencies were related to weekly external load training volume (accumulated weekly loads). The authors did not consider external match load measures relativized per minute. Overall, per-minute measures are expected to vary significantly in congested fixtures due to accumulated fatigue caused by the lack of recovery time given to athletes playing in three matches in a week. For those reasons, we hypothesize that some measures per minute may present within-week and within-match variations, while higher high-intensity measures such as maxspeed may remain consistent.

Following the above-mentioned statements and in an attempt to provide better insights to coaches and practitioners regarding the characterization and the impact that playing in three matches in a row during one week has on soccer players, this study aimed to (i) analyze variations in distance covered $(\mathrm{m} / \mathrm{min})$, high-speed running $(\mathrm{m} / \mathrm{min})$, high-metabolic load distance (A.U./min), and maximal speed $(\mathrm{m} / \mathrm{s}$ ) over three consecutive matches within a congested week and (ii) analyze within-match variations (between halves) in external load measures during congested weeks.

\section{Methods}

\subsection{Experimental approach to the problem}

This study followed an observational cohort design. Elite soccer players were monitored throughout a full season (from July 3, 2018 to May 9, 2019). Only the 45 weeks of in-season training and competition period was analyzed considering the purpose of analyzing only congested weeks. Congested weeks were classified as those in which three matches were played. Only players who participated for at least 45 minutes in all three matches during a congested week were considered for the analysis. External loads were monitored daily using an 18-Hz GPS. The monitoring process occurred during home and away matches, all of which took place in the same country (England).

\subsection{Participants}

The cohort included nineteen professional male players (age: $26.5 \pm 4.3$ years; height: $180.2 \pm 7.3 \mathrm{~cm}$; body mass: $75.6 \pm$ $9.6 \mathrm{~kg}$; experience as professionals: $7.5 \pm 4.3$ years) from an English Premier First League team voluntarily participated in this study. The eligibility criteria for being considered in the analysis were: (i) participation in at least two consecutive matches (i.e., 1st and 2nd OR 2nd and 3rd OR 1st, 2nd, and 3rd); (ii) absence of injury or illness during the congested weeks and the three weeks before them; (iii) absence of any injury lasting more than four consecutive weeks through- 
Week 1

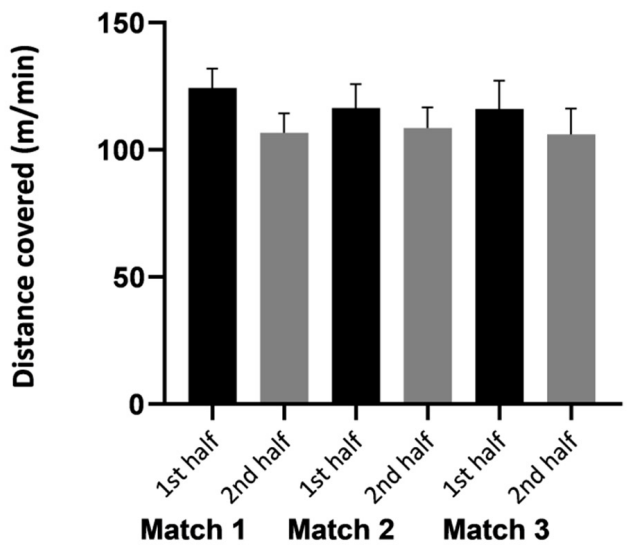

Week 1

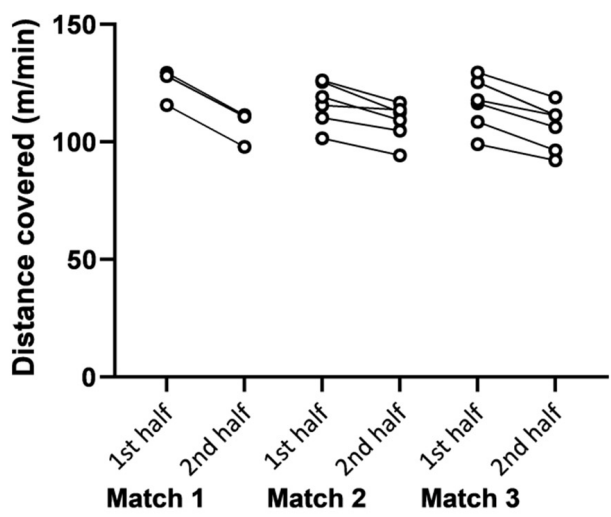

F IG. 1. Mean and SD of distance covered $(\mathrm{m} / \mathrm{min})$ in the $1 \mathrm{st}$ and $2 \mathrm{nd}$ half of three consecutive matches for Week 1.

Week 2

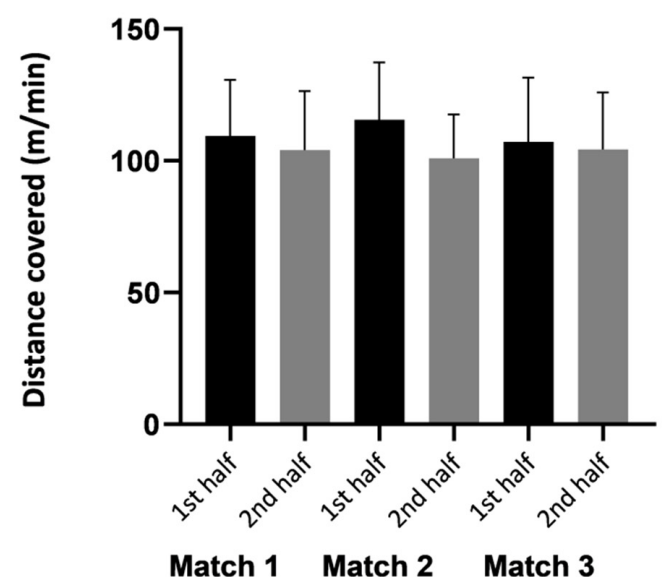

Week 2

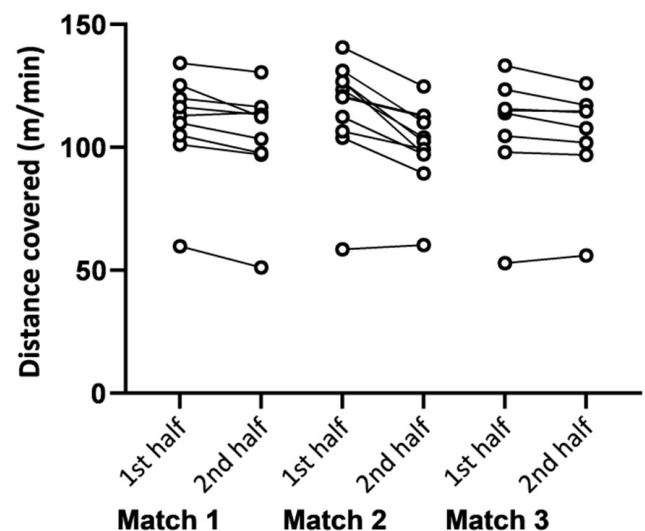

F IG. 2. Mean and SD of distance covered ( $\mathrm{m} / \mathrm{min})$ in the 1st and $2 \mathrm{nd}$ half of three consecutive matches for Week 2.

out the season. From the 25 players initially included, 6 players were excluded because they had not participated in two consecutive matches. Detailed information about the study design, procedures, and methodological approach was provided before the beginning of the study, and all players signed a free informed consent form before participating. The study followed the ethical standards of the Declaration of Helsinki and was approved by the scientific council of the local university.

\subsection{External load quantification}

The players were monitored with an $18-\mathrm{Hz}$ GPS unit (STATSports, Apex, Newry, Northern Ireland) during the study. The employed GPS revealed good levels of accuracy and variability at different speed thresholds [22] and excellent inter-unit reliability for peak velocity [23]. Considering the current issues related to GPS distance-based measurements (mainly accelerometry-based measures), the use of $18-\mathrm{Hz}$ GPS sampling shows greater validity and reliability than 10$\mathrm{Hz}$ and $15-\mathrm{Hz}$ samplings [24]. While 18-Hz GPS sampling and $20-\mathrm{Hz}$ local positioning systems (LPSs) have greater validity and reliability than $10-\mathrm{Hz}$ and $15-\mathrm{Hz}$ GPSs, they also present greater measurement errors [25].

The units were placed in a specific vest fixed between the scapulae, and each player used the same unit throughout the season to reduce inter-variability. During the period of data collection, the range of satellites was 18 to 21 . The data collected during matches were imported and processed in the STATSports APEX software (version 5.0) [23]. The following measures were extracted from each match: (i) total distance (TD: the total distance covered by players); (ii) high-speed running (HSR: distances covered at a speed of $19.8 \mathrm{~W} \cdot \mathrm{h}^{-1}$ or above); (iii) high-metabolic load distance (HMP: distances covered at HSR, coupled with accelerations and decelerations at a magnitude of $2 \mathrm{~m} / \mathrm{s}^{2}$ or above); and (iv) maximal speed (maxSpeed: maximal running velocity reached). All the selected GPS-derived measures were relativized to match duration by dividing each metric value per minute. 
Week 3

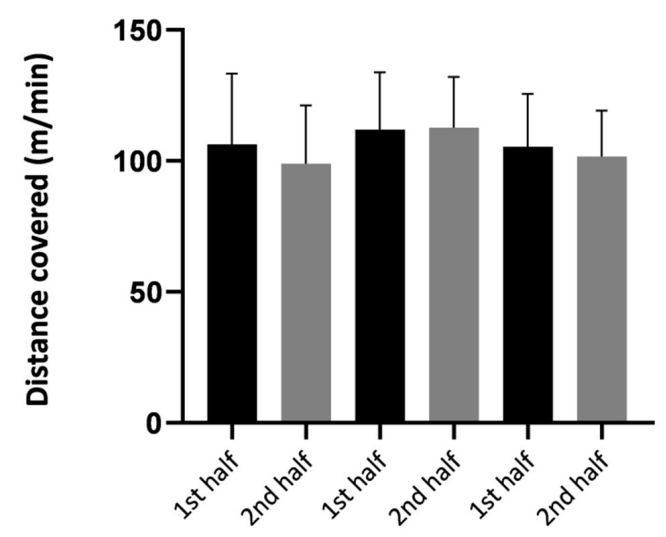

Match 1 Match 2 Match 3

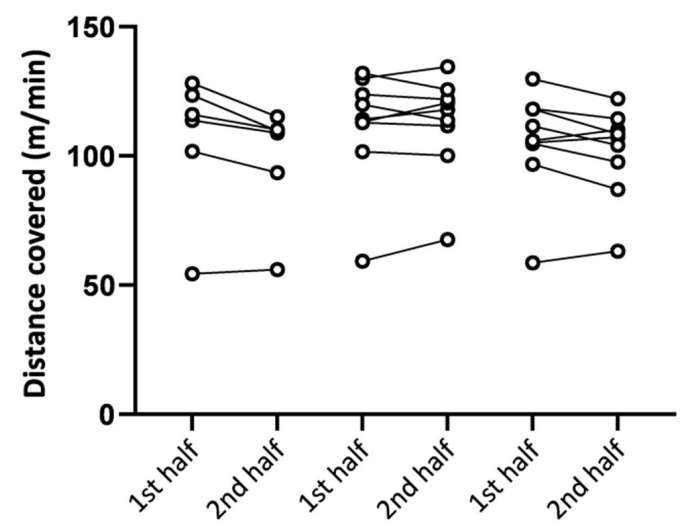

Match 1 Match 2 Match 3

F IG. 3. Mean and SD of distance covered $(\mathrm{m} / \mathrm{min})$ in the 1st and $2 \mathrm{nd}$ half of three consecutive matches for Week 3.

\subsection{Statistical procedures}

Data analysis was performed using the SPSS software version 26.0 (SPSS, Inc., Chicago, IL, USA) program. Data were expressed as mean \pm standard deviation (SD) in bar graphs, and individual variations in three consecutive matches for each week were shown in scatter plot graphs. Two-way repeated measures ANOVA was used to analyze the variations of external load measures (distance covered, high-speed running, high- metabolic power distance and maximal speed) measured in two halves (1st and 2nd half) according to three consecutive matches (match 1, match 2, match 3) for each week (week 1, 2 and 3). Variances were found to be homogeneous for all external load measures. Therefore, sphericity assumed values were taken into account $(p>0.05)$. The Bonferroni test was used to make the pairwise differences. In addition, the standardized effect size (ES) of Cohen's d was calculated for pairwise comparisons. The Ferguson's classification was used to evaluate the ES as follows [26]: no effect $(\mathrm{ES}<0.04)$, minimum effect $(0.04 \leq \mathrm{ES}<0.25)$, moderate effect $(0.25 \leq \mathrm{ES}<0.64)$, and strong effect (ES $\geq 0.64$ ). Significance level was interpreted according to $p<$ $0.05, p<0.1$ and $p<0.001$.

\section{Results}

The Fig. 1 shows the differences between halves of the three consecutive matches played in week 1 , for distance covered.

A statistically significant difference was found in the comparison of distance covered of 1 st half and 2 nd half mean values regardless of matches $\left(\mathrm{F}_{(1,12)}=188.398, p<0.01\right.$, partial eta squared: 0.940). According to Bonferroni post-hoc analyses, there was a significant decrease in distance covered in the 2 nd half versus the 1 st half (standardized difference ES: $1.12(0.59 ; 1.65,95 \% \mathrm{CI})$, strong effect). Matches and halves interaction revealed a significant effect on distance covered $\left(\mathrm{F}_{(2,12)}=9.903, p<0.01\right.$, partial eta squared: 0.623$)$. There was greater decrease in distance covered in the first match (standardized difference ES: 2.31 (-2.66; 7.30, 95\% CI), strong effect) as compared with the second and third matches
(Second match: standardized difference ES: 0.83 (0.02; 1.64, 95\% CI), strong effect; Third match: standardized difference ES: $0.88(-0.11 ; 1.65,95 \% \mathrm{CI})$, strong effect).

The Fig. 2 shows the differences between halves of the three consecutive matches played in week 2 , for distance covered.

In the second week, significant difference was observed in the comparison of distance covered 1 st half and 2 nd half without any match distinction $\left(\mathrm{F}_{(1,25)}=39.012, p<0.01\right.$, partial eta squared: 0.609). A significant decrease was observed for distance covered in the 2 nd half compared to the 1 st half (standardized difference ES: 0.38 (0.20; 0.56 , 95\% CI), moderate effect). Matches and halves interaction showed a statistically significant effect on the distance covered $\left(\mathrm{F}_{(2,25)}=9.357, p<0.001\right.$, partial eta squared: 0.428$)$. There was greater decrease in distance covered in the second match (standardized difference ES: 0.63 (0.22; 1.04, 95\% $\mathrm{CI}$ ), moderate effect) than in the first and third match (First match: standardized difference ES: 0.23 (0.04; 0.42, 95\% CI), minimum effect; Third match: standardized difference ES: 0.07 (-0.01; 0.16, 95\% CI), minimum effect).

The Fig. 3 shows the differences between halves of the three consecutive matches played in week 3 , for distance covered.

In the third week, a significant decrease in distance covered was found in the 2 nd half compared to the 1 st half regardless of the three consecutive matches $\left(\mathrm{F}_{(1,21)}=8.717, p<0.01\right.$, partial eta squared: 0.293 , standardized difference ES: 0.13 (0.00; 0.26), minimum effect). Matches and halves interaction on distance covered was statistically significant $\left(\mathrm{F}_{(2,21)}=\right.$ 3.827, $p<0.05$, partial eta squared: 0.267$)$. There was greater decrease in distance covered in the first match (standardized difference ES: 0.16 (-0.02; 0.36, 95\% CI), minimum effect) compared to the second and third match. (Second match: standardized difference ES: $-0.03(-0.21 ; 0.15,95 \% \mathrm{CI})$, no effect; Third match: standardized difference ES: 0.18 (-0.05; $0.42,95 \% \mathrm{CI})$, minimum effect). 
Week 1

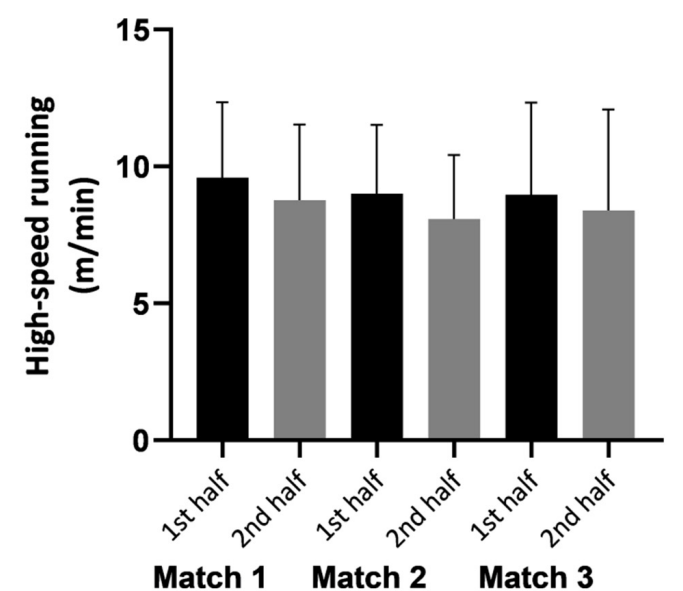

Week 2

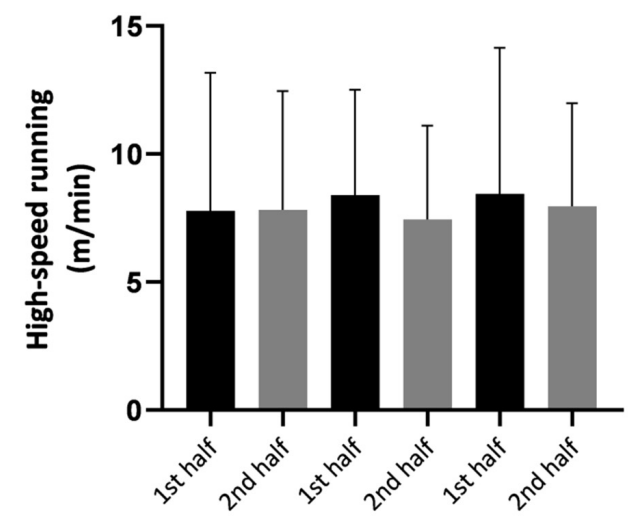

Match 1 Match 2 Match 3
Week 1

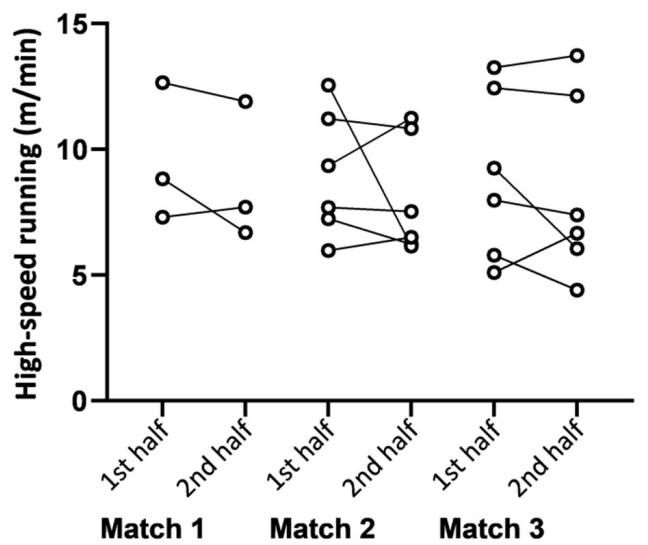

Week 2

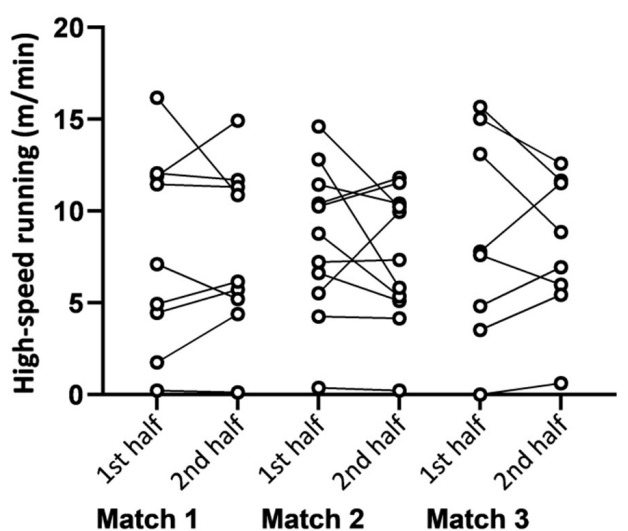

F IG. 4. Mean and SD of high-speed running $(\mathrm{m} / \mathrm{min})$ in the 1 st and 2 nd half of three consecutive matches for Week 1 and Week 2.

The Fig. 4 shows the differences between halves of the three consecutive matches played in week 1 and 2, for highspeed running.

No significant difference was found in the comparison of 1 st half and 2 nd half mean values of high-speed running performance without discriminating matches (Week 1: $\mathrm{F}_{(1,12)}=$ 1.708, $p>0.05$, partial eta squared: 0.125 , standardized difference ES: $0.27(-0.14 ; 0.69)$, moderate effect; Week 2: $\mathrm{F}_{(1,25)}=0.710, p>0.05$, partial eta squared: 0.028 , standardized difference ES: 0.10 ( $-0.13 ; 0.34)$, minimum effect). Matches and halves interaction had no statistically significant effect on high-speed running performance (Week 1: $\mathrm{F}_{(2,12)}=$ $0.041, p>0.05$, partial eta squared: 0.007 , Week $2: \mathrm{F}_{(2,25)}=$ $0.281, p>0.05$, partial eta squared: 0.022 ).

The Fig. 5 shows the differences between halves of the three consecutive matches played in week 3 , for high-speed running.

In the third week, there was a significant difference in high-speed running in the $2 \mathrm{nd}$ half compared to the $1 \mathrm{st}$ half $\left(\mathrm{F}_{(1,12)}=7.035, p>0.05\right.$, partial eta squared: 0.251 , standardized difference ES: $0.41(0.08 ; 0.73,95 \% \mathrm{CI})$, moderate effect). Moreover, Matches and halves interaction did not significantly had an impact on high-speed running perfor- mance $\left(F_{(2,21)}=0.083, p>0.05\right.$, partial eta squared: 0.008).

The Fig. 6 shows the differences between halves of the three consecutive matches played in week 1, for high-metabolic load distance.

In the first week, high-metabolic load distance decreased in the 2 nd half compared to the 1st half, regardless of the three consecutive matches $\left(\mathrm{F}_{(1,12)}=116.594, p<0.01\right.$, partial eta squared: 0.907 , standardized difference ES: $0.89(0.43 ; 1.35$, 95\% CI), strong effect). In addition, Matches and halves interaction was found to have a significant effect on highmetabolic load distance $\left(\mathrm{F}_{(2,12)}=8.861, p<0.01\right.$, partial eta squared: 0.596). There was greater decrease in highmetabolic load distance in the first match (standardized difference ES: $1.39(-1.75 ; 4.53,95 \% \mathrm{CI})$, strong effect) than in the other matches (Second match: standardized difference ES: 0.84 (0.01; 1.67, 95\% CI), strong effect; Third match: standardized difference ES: $0.64(0.06 ; 1.22,95 \% \mathrm{CI})$, strong effect).

The Fig. 7 shows the differences between halves of the three consecutive matches played in week 2, for high-metabolic load distance.

In the second week, there was significant difference between 1 st half and 2 nd half for high-metabolic load dis- 
Week 3

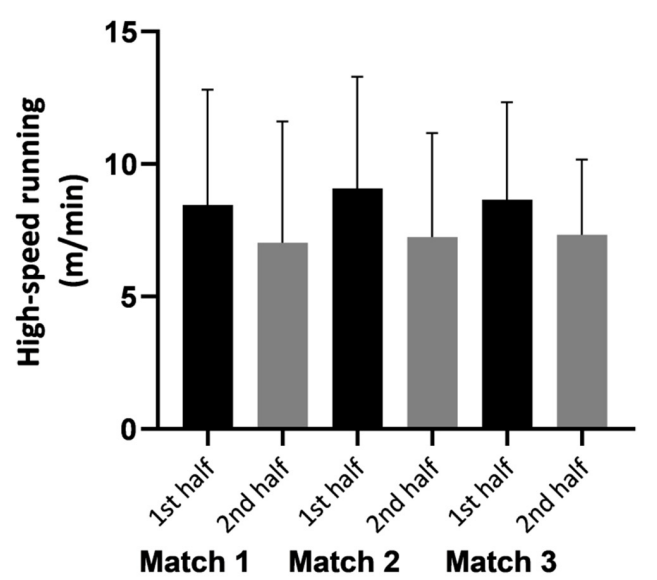

Week 3

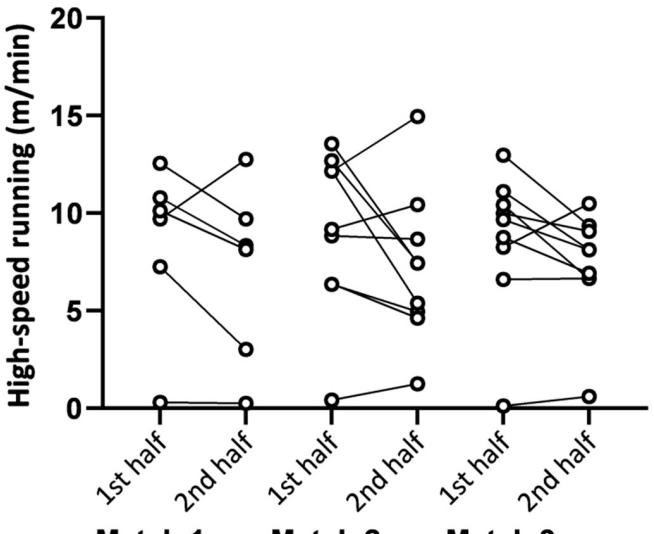

Match 1 Match 2 Match 3

F IG. 5. Mean and SD of high-speed running $(\mathrm{m} / \mathrm{min})$ in the $1 s t$ and 2 nd half of three consecutive matches for Week 3.

Week 1

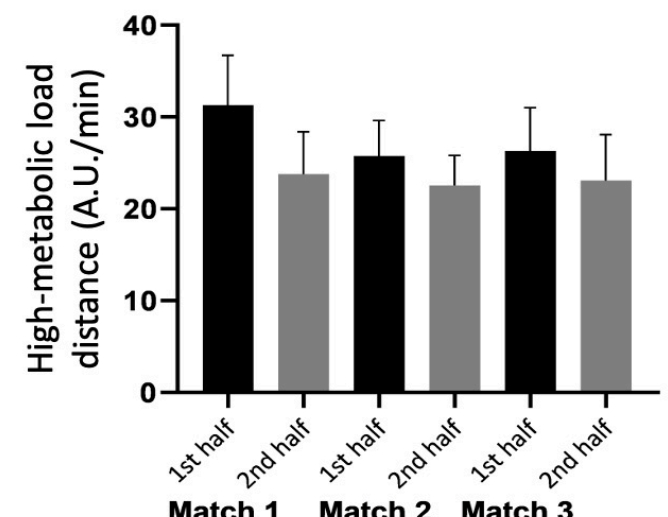

Week 1

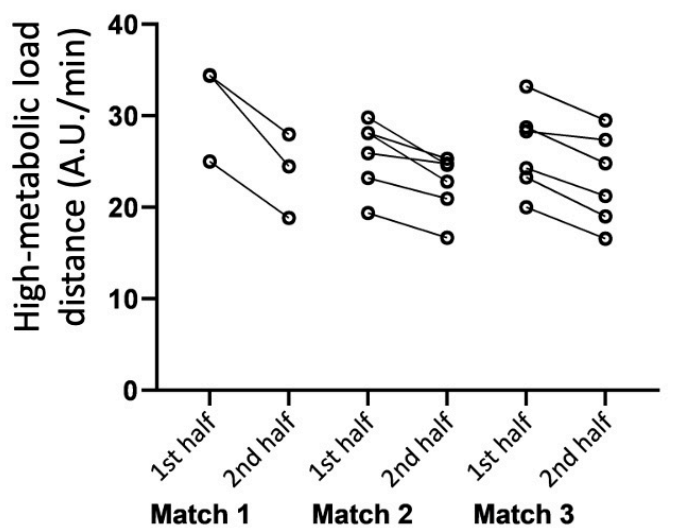

F IG. 6. Mean and SD of high-metabolic load distance (A.U./min) in the 1st and $2 \mathrm{nd}$ half of three consecutive matches for Week 1 .

Week 2

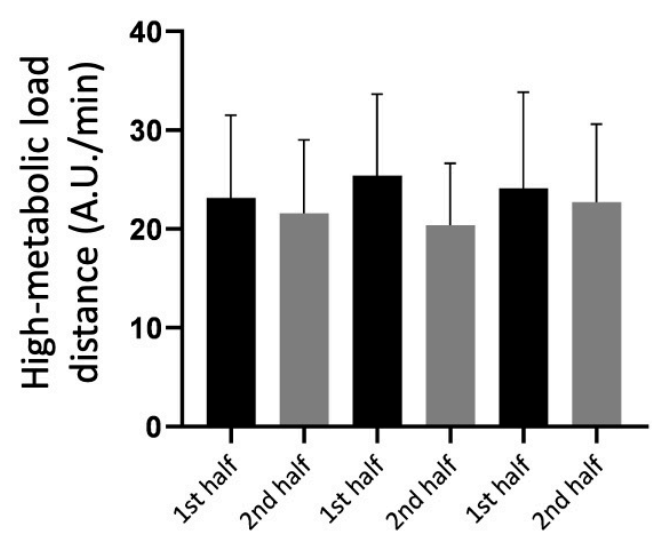

Match 1 Match 2 Match 3
Week 2

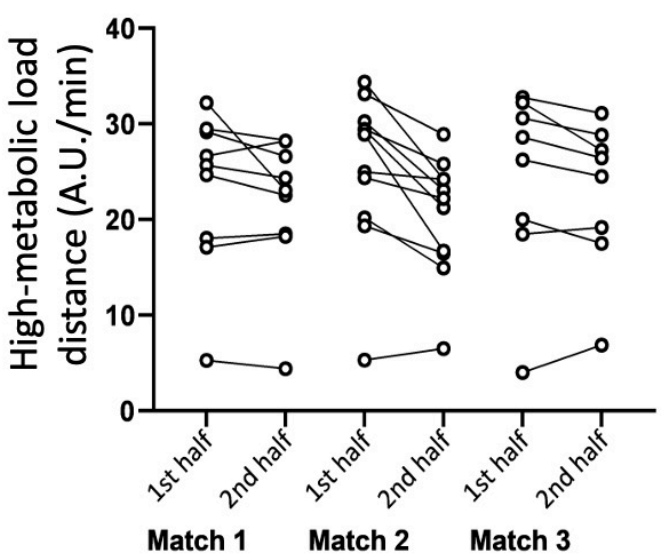

F IG. 7. Mean and SD of high-metabolic load distance (A.U./min) in the 1st and 2 nd half of three consecutive matches for Week 2.

tance without any matches distinction ( $p<0.01$, partial eta squared: 0.409). High-metabolic load distance decreased in the 2 nd half as compared with 1 st half (standardized difference ES: $0.34(0.14 ; 0.53,95 \% \mathrm{CI})$, moderate effect). Matches and halves interaction had a significant effect on metabolic power $\left(\mathrm{F}_{(2,25)}=3.721, p<0.05\right.$, partial eta squared: 0.229$)$. The high-metabolic load distance in the second match (standardized difference ES: $0.61(0.16 ; 1.06,95 \% \mathrm{CI})$, moderate 
Week 3

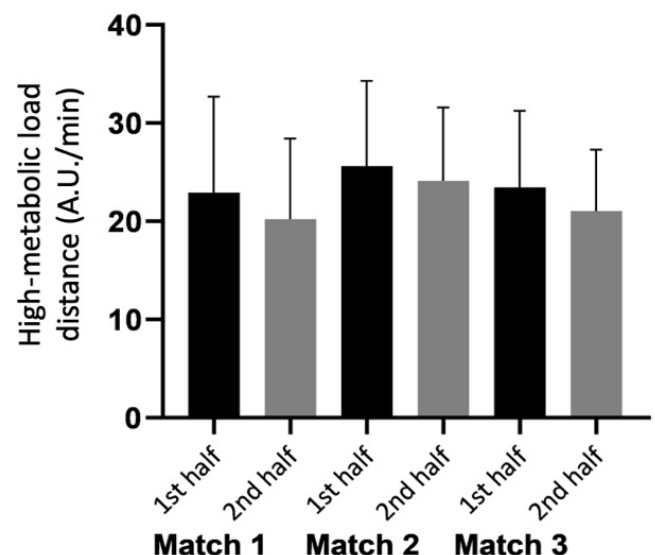

Week 3

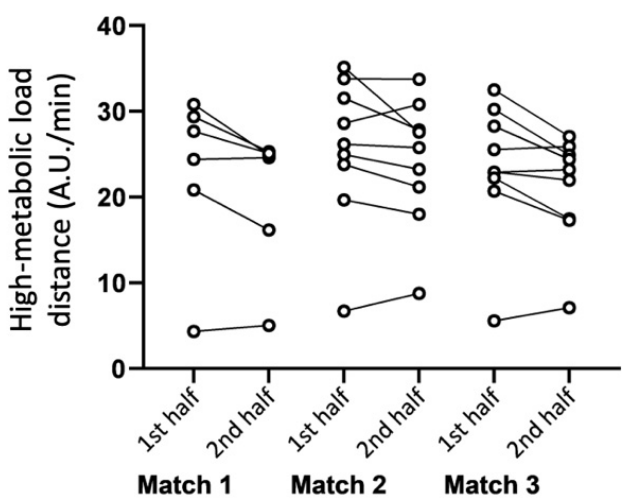

F IG. 8. Mean and SD of high-metabolic load distance (A.U./min) in the 1st and 2 nd half of three consecutive matches for Week 3.

Week 1

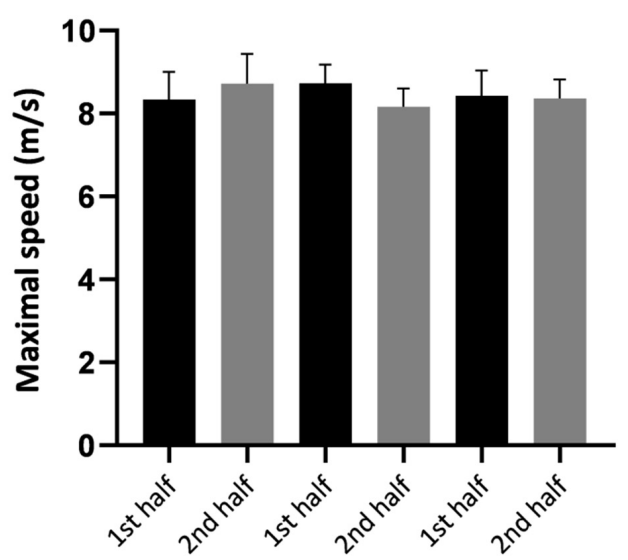

Match 1 Match 2 Match 3
Week 1

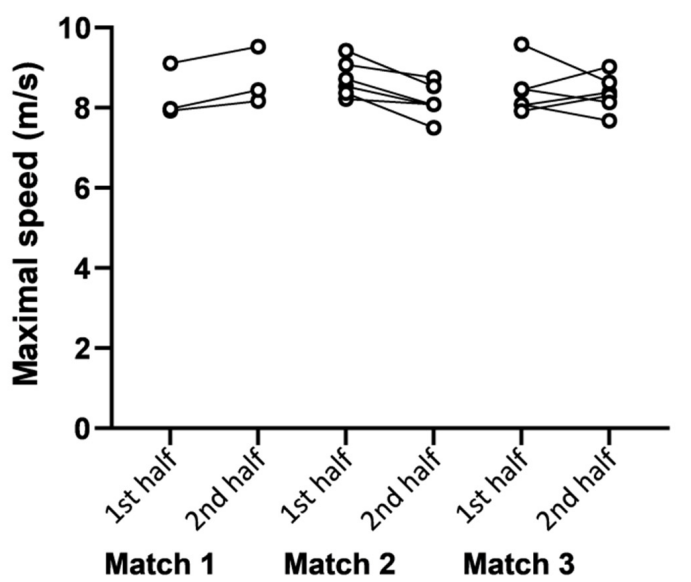

F IG. 9. Mean and SD of maximal speed $(\mathrm{m} / \mathrm{s})$ in the 1 st and $2 \mathrm{nd}$ half of three consecutive matches for Week 1.

effect) showed significantly greater decrease compared to the first and third match (First match: standardized difference ES: 0.18 (-0.12; 0.50, 95\% CI), minimum effect; Third match: standardized difference ES: $0.09(-0.05 ; 0.25,95 \% \mathrm{CI})$, minimum effect).

The Fig. 8 shows the differences between halves of the three consecutive matches played in week 3, for high-metabolic load distance.

In the third week, high-metabolic load distance showed a decrease in 2 nd half versus in 1 st half without discriminating three consecutive matches $\left(\mathrm{F}_{(1,21)}=14.066, p<0.01\right.$, partial eta squared: 0.401 , standardized difference ES: 0.25 (0.09; $0.40,95 \% \mathrm{CI})$, moderate effect). The effect of Matches and halves interaction on high-metabolic load distance was not found to be statistically significant $\left(\mathrm{F}_{(2,21)}=0.392, p>0.05\right.$, partial eta squared: 0.036).

The Fig. 9 shows the differences between halves of the three consecutive matches played in week 1 , for maximal speed.

In the first week, no significant differences in maximal speed were found between 1 st half and $2 \mathrm{nd}$ half, independent of the matches $\left(\mathrm{F}_{(1,12)}=0.505, p>0.05\right.$, partial eta squared: 0.040, standardized difference ES: 0.33 (-0.25; 0.91, 95\% CI), moderate effect). Matches and halves interaction on maximal speed was found to be statistically significant $\left(\mathrm{F}_{(2,12)}=5.044\right.$, $p<0.05$, partial eta squared: 0.457). The maximal speed decreased in the 2 nd half compared to the 1 st half in second (standardized difference ES: $1.25(0.01 ; 2.49,95 \% \mathrm{CI})$, strong effect) and third match (standardized difference ES: 0.12 ($1.01 ; 1.26,95 \% \mathrm{CI})$, minimum effect). The maximal speed increased in the 2 nd half versus the 1 st half in first match (standardized difference ES: $-0.49(-1.62 ; 0.63$, 95\% CI), moderate effect).

The Fig. 10 shows the differences between halves of the three consecutive matches played in week 2, for maximal speed.

In the second week, there was no significant difference in maximal speed between 1 st half and 2nd half, without discriminating the matches $\left(\mathrm{F}_{(1,25)}=3.509, p>0.05\right.$, partial eta squared: 0.123$)$. Despite not significant, the maximal 
Week 2

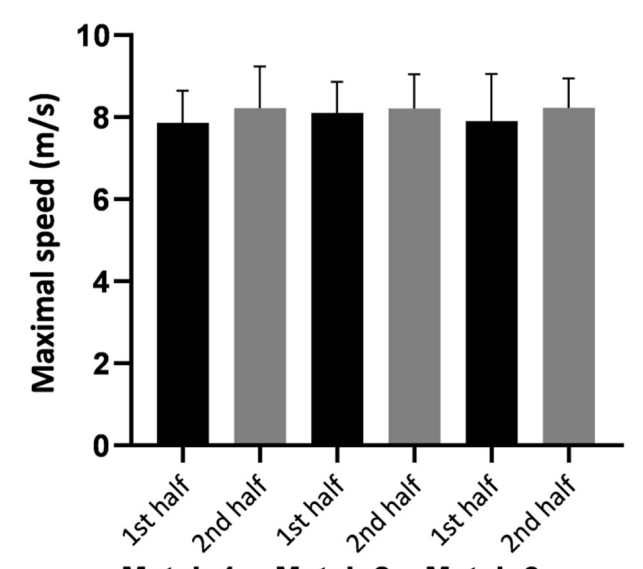

Match 1 Match 2 Match 3

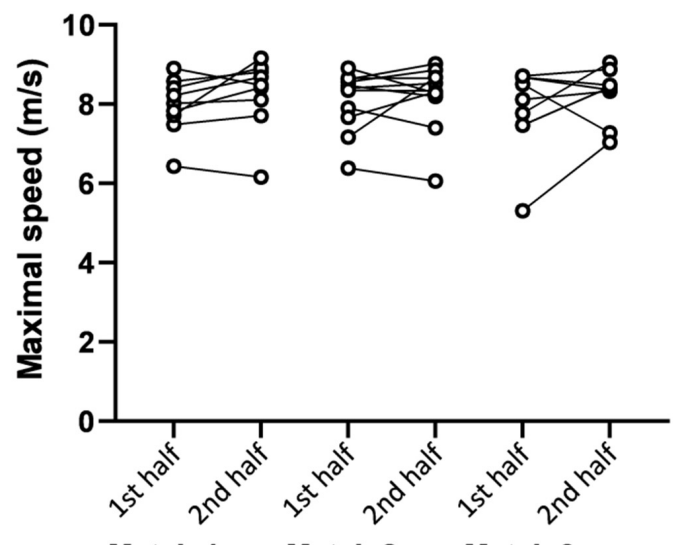

Match 1 Match 2 Match 3

F I G. 10. Mean and SD of maximal speed (m/s) in the 1st and 2nd half of three consecutive matches for Week 2.

Week 3

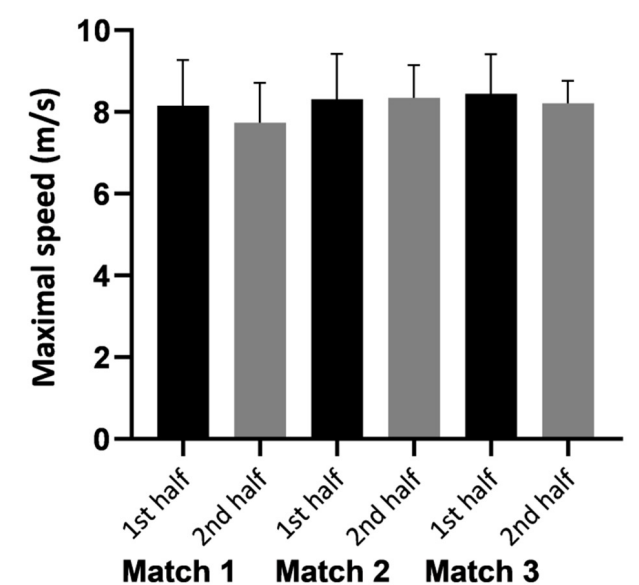

Week 3

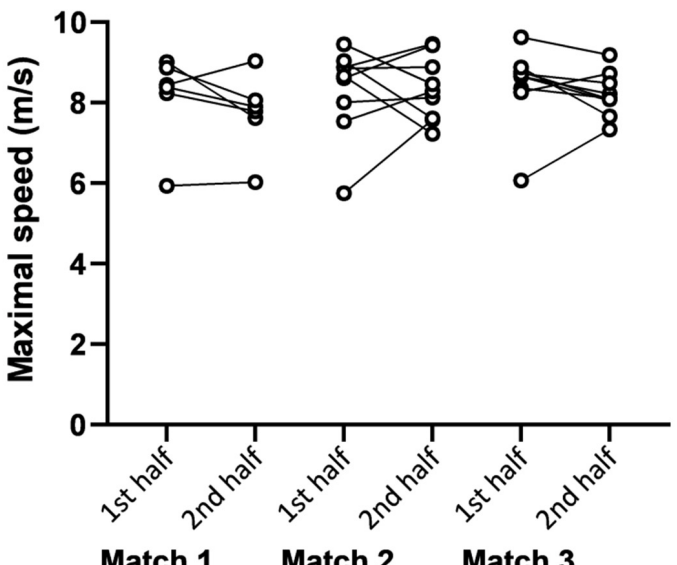

Match 1 Match 2 Match 3

F IG. 11. Mean and SD of maximal speed $(\mathrm{m} / \mathrm{s})$ in the 1 st and $2 \mathrm{nd}$ half of three consecutive matches for Week 3.

speed was higher in the 2 nd half than in the 1 st half (standardized difference ES: $-0.29(-0.62 ; 0.04,95 \%$ CI $)$, moderate effect). Moreover, Matches and halves interaction did not have a significant effect on maximal speed $\left(\mathrm{F}_{(2,25)}=0.291, p\right.$ $>0.05$, partial eta squared: 0.023$)$. There was an increase in maximal speed in the 2 nd half compared to the 1 st half in each match (First match: standardized difference ES: -0.36 (-0.90; $0.16,95 \% \mathrm{CI})$, moderate effect; Second match: standardized difference ES: -0.14 (-0.65; 0.37, 95\% CI), minimum effect; Third match: standardized difference ES: $-0.32(-1.13 ; 0.49$, 95\% CI), moderate effect).

The Fig. 11 shows the differences between halves of the three consecutive matches played in week 3 , for maximal speed.

In the third week, the maximal speed value was not statistically significant different between the 1 st half and 2 nd half $\left(\mathrm{F}_{(1,21)}=1.225, p>0.05\right.$, partial eta squared: 0.055$)$. Despite not significant, the maximum speed decreased by the 2 nd half compared to the 1 st half (standardized difference ES: 0.19 (-
0.20; 0.59, 95\% CI), minimum effect). In addition, Matches and halves interaction did not significantly have an impact on maximal speed $\left(\mathrm{F}_{(1,21)}=0.476, p>0.05\right.$, partial eta squared: $0.043)$. The maximum speed showed a decrease by the 2 nd half compared to the 1 st half in the first and third match (First match: standardized difference ES: 0.38 (-0.36; 1.12, 95\% CI), moderate effect; Third match: standardized difference ES: $0.26(-0.37 ; 0.89,95 \% \mathrm{CI})$, moderate effect).

\section{Discussion}

This study aimed to analyze within-match (betweenhalves) and within-week variations in TD, HSR, HML, and maxSpeed intensities $(\mathrm{m} / \mathrm{min})$ during congested weeks among male soccer players. The main findings revealed that TD and HML measures (relativized per minute) show meaningful within-week and within-match differences, while HSR and maxSpeed remain unchanged. 
TD comparisons revealed significant TD decreases during the second halves of matches in all weeks. Also, greater between-halves TD decreases were seen in Match 1 than in subsequent matches in week 1 and week 3. Meanwhile, Match 2 presented the greatest TD decrease in week 2 . A recent systematic review that analyzed the effects of congested fixtures on external load performance affirmed that TD presents no significant differences during congested weeks containing two to three matches [15]. Indeed, a greater TD volume in Match 3 was shown during a congested week in previous research, suggesting that TD is not affected during congested weeks. This is similar to our findings regarding TD match intensity.

A study conducted on 11 elite soccer players revealed that TD was greater during the first half of a match than in the second half [27], which corroborates our results in terms of intensity. However, the same authors [27] suggested that physical and skill performance were not affected after a week containing three matches. It could be that TD intensities declined steadily over the course of the three matches. However, certain contextual factors, such as player rotation, opposition quality, and players' pacing strategies to reduce running volumes, might have affected these findings [28, 29].

Considering HSR intensity, our results showed no significant differences between the first and second halves of games in week 1 and week 2, regardless of the number of matches played. Meanwhile, significant differences were observed in this regard in week 3 . In fact, it was previously documented that high-intensity running distances covered in elite soccer are not significantly different across three matches played in the same week [30]. Although that study considered HSR volume and not intensity, the findings were similar to the results of the present study. Specifically, they suggest that HSR intensities are unaffected during congested weeks containing three matches.

In contrast, another study conducted on 11 elite soccer players revealed a progressive decrease in HSR distances during a congested week with three matches [31]. However, the two above-mentioned studies [30, 31] used different speed thresholds to measure HSR, which might account for the significant differences in the outcomes and subsequent comparisons. HSR is a measure of great importance given its relationship with lower-body injuries, especially hamstring muscle injuries $[32,33]$. Therefore, understanding the impacts of HSR volume and intensity during congested weeks is of paramount importance to allow coaches to adjust training sessions accordingly.

Regarding the HML measure, significant decreases were reported during second halves in all weeks, regardless of the number of matches. HML is an important metric, as it corresponds to distances covered above $25.5 \mathrm{~W} \cdot \mathrm{kg}^{-1}$ of metabolic power, which is an accurate indicator of energetic cost [34, 35]. There is a lack of evidence supporting the effects of congested fixtures on HML distances. One recent study conducted on 19 elite soccer players revealed that players who participated in three matches during congested weeks presented greater weekly HML distances than those who played in one or two matches [20]. The same authors suggested that HML is affected by congested fixtures, which aligns with our results [20]. However, the mentioned study considered weekly HML volume, while the present study considered HML match intensity only [20]. Another study aiming to analyze HML variations between match halves on 21 soccer players revealed significant HML decreases with moderateto-high effect sizes in the second half, which also supports our findings. This suggests that HML volume and intensity present similarly significant decreases in second halves of matches during congested and non-congested weeks.

Regarding the maxSpeed measure, no significant differences were found between halves for all weeks, regardless of the number of matches. Few studies have reported maxSpeed variations across three matches played during a single week. However, one previous study indicated that high-intensity running-including maxSpeed-did not change during congested weeks [18]. This is in line with our results, as we observed slight increases during the second halves of some matches in week 1 and week 2. Although studies have focused on these types of metric volumes [36], the present study suggests that maxSpeed intensity is not affected by participation in three matches in one week. Also, no differences were observed between match halves. Measuring athletes' maximal sprinting speeds is of paramount importance to adjusting training sessions and avoiding injuries [37]. Although maxSpeed intensity seems to remain unchanged within matches and within congested weeks, coaches should consider weekly sprinting distance recommendations to ensure that athletes are resilient enough to tolerate such loads in congested weeks [33].

Based on the evidence presented in the current study, the volume and intensity of the analyzed measures appear to present similar patterns across three-match congested weeks. Coaches and practitioners should analyze the within-match and within-week variations of different GPS metrics' intensities-especially TD and HML measures per minute-to ensure that training is adjusted appropriately to suit players who participate in multiple matches during congested weeks. Also, analyzing variations in external loads (relativized per-minute using effect sizes) during congested weeks could help coaches track meaningful changes.

This study was not without its limitations. One of the main limitations was the small sample size, which is a typical issue in studies conducted in elite team sports settings. Another limitation was that the results from congested weeks were not compared with those from non-congested weeks. Furthermore, changes in performance across playing positions were not considered in the present study-however, this was beyond the scope of the present study.

Also, this study suffered from some issues related to the use of GPS systems [24]. Some GPS measures, especially distance-based measures, can depend heavily on contextual factors, such as winning or losing. Such dependencies could have influenced our findings [38, 39]. Finally, the fitness levels of players were not measured or considered as possible covariables. These attributes might play an important role 
in the recovery or the ability to delay the effects of fatigue. Future studies should utilize larger samples to reveal generalized patterns of load intensities while considering the contextual factors that might affect physical performance during congested weeks. Additionally, fitness parameters should be collected and considered as covariables.

\section{Conclusions}

The current study revealed that TD and HML present significant differences between both match halves in the three analyzed weeks. Meanwhile, HSR and maxSpeed measures exhibited no significant overall differences across matches. For these reasons, coaches and practitioners should consider the effects of congested weeks on TD and HML distances to ensure that players can cope with the energetic cost associated with participating in three consecutive matches in one week. Also, even though higher-intensity metrics such as HSR and maxSpeed intensities seem to remain unchanged during congested weeks, they still might need to be adjusted according to each player's needs.

\section{Author contributions}

Conceptualization, RS and FMC; methodology, RS, FMC and BM; formal analysis, RS; investigation, FMC and BM; writing-original draft preparation RS, HC, SAC, TS, BM and FMC; writing-review and editing, RS, HC, SAC, TS, BM, GB and FMC. All authors have read and agreed to the published version of the manuscript.

\section{Ethics approval and consent to participate}

The players were previously informed about the study design and procedures. After receiving the information, the players signed a free consent about his participation in the study. Despite the monitoring process belonging to the daily routine in the club, they made it voluntarily. The ethical standards of the updated Declaration of Helsinki (2013 version) were ensured during the study. The study was approved by the scientific council of the School of Sport and Leisure (ID: 2018ESDL031).

\section{Acknowledgment}

This study made part of one curricular unit of Master in Sports Training at Escola Superior de Desporto e Lazer, Instituto Politécnico de Viana do Castelo, Portugal.

\section{Funding}

This study received no external funding.

\section{Conflict of interest}

The authors declare no conflict of interest.

\section{References}

[1] Travassos B, Davids K, Araújo D, Esteves PT. Performance analysis in team sports: Advances from an Ecological Dynamics approach. International Journal of Performance Analysis in Sport. 2013; 13: 8395.

[2] Clemente F, Silva R, Arslan E, Aquino R, Castillo D, Mendes B. The effects of congested fixture periods on distance-based workload indices: A full-season study in professional soccer players. Biology of Sport Vols. 2021; 38: 37-44.

[3] Hader K, Rumpf MC, Hertzog M, Kilduff LP, Girard O, Silva JR. Monitoring the Athlete Match Response: can External Load Variables Predict Post-match Acute and Residual Fatigue in Soccer? A Systematic Review with Meta-analysis. Sports Medicine Open. 2019; 5: 48 .

[4] Gabbett T. The Training-Performance Puzzle: how can the Past Inform Future Training Directions? Journal of Athletic Training. 2020; 55: 874-884.

[5] Nédélec M, McCall A, Carling C, Legall F, Berthoin S, Dupont G. Recovery in soccer: part ii-recovery strategies. Sports Medicine. 2013; 43: 9-22.

[6] Impellizzeri FM, Marcora SM, Coutts AJ. Internal and External Training Load: 15 Years on. International Journal of Sports Physiology and Performance. 2019; 14: 270-273.

[7] Gabbett T, Nassis GP, Oetter E, Pretorius J, Johnston N, Medina D, et al. The athlete monitoring cycle: a practical guide to interpreting and applying training monitoring data. British Journal of Sports Medicine. 2017; 51: 1451-1452.

[8] Malone J, Lovell R, Varley MC, Coutts AJ. Unpacking the Black Box: Applications and Considerations for Using GPS Devices in Sport. International Journal of Sports Physiology and Performance. 2017; 12: S218-S226.

[9] Akenhead R, Nassis GP. Training Load and Player Monitoring in High-Level Football: Current Practice and Perceptions. International Journal of Sports Physiology and Performance. 2017; 11: 587-593.

[10] Nédélec M, McCall A, Carling C, Legall F, Berthoin S, Dupont G. Recovery in Soccer. Sports Medicine. 2012; 42: 997-1015.

[11] Rojas-Valverde D, Gutiérrez-Vargas R, Rodríguez-Montero A, Pereira LA, Loturco I, Martín-Rodríguez S. Reduced muscle contractile function in elite young soccer players after a shortcongested fixture period. Proceedings of the Institution of Mechanical Engineers. 2019; 233: 249-257.

[12] Wollin M, Thorborg K, Pizzari T. Monitoring the effect of football match congestion on hamstring strength and lower limb flexibility: Potential for secondary injury prevention? Physical Therapy in Sport. 2018; 29: 14-18.

[13] Lundberg TR, Weckström K. Fixture congestion modulates postmatch recovery kinetics in professional soccer players. Research in Sports Medicine. 2018; 25: 408-420.

[14] Silva JR, Rumpf MC, Hertzog M, Castagna C, Farooq A, Girard O, et al. Acute and Residual Soccer Match-Related Fatigue: a Systematic Review and Meta-analysis. Sports Medicine. 2018; 48: 539-583.

[15] Julian R, Page RM, Harper LD. The Effect of Fixture Congestion on Performance during Professional Male Soccer Match-Play: a Systematic Critical Review with Meta-Analysis. Sports Medicine. 2021; 51: 255-273.

[16] Springham M, Williams S, Waldron M, Strudwick AJ, Mclellan C, Newton RU. Prior workload has moderate effects on high-intensity match performance in elite-level professional football players when controlling for situational and contextual variables. Journal of Sports Sciences. 2020; 38: 2279-2290.

[17] Carling C, Gall F, Dupont G. Are physical performance and injury risk in a professional soccer team in match-play affected over a prolonged period of fixture congestion? International Journal of Sports Medicine. 2012; 33: 36-42.

[18] Djaoui L, Wong DP, Pialoux V, Hautier C, Da Silva CD, Chamari $\mathrm{K}$, et al. Physical Activity during a Prolonged Congested Period in a top-Class European Football Team. Asian Journal of Sports Medicine. 2014; 5: 47-53. 
the match? The International Journal of Sports Science \& Coaching. 2021; 1-10.

[30] Soroka A, Lago-Peñas C. The effect of a succession of matches on the physical performance of elite football players during the World Cup Brazil 2014. International Journal of Performance Analysis in Sport. 2016; 16: 434-441.

[31] Andrzejewski M, Konarski MJ, Chmura J, Pluta B. Changes in the activity profiles of soccer players over a three-match training micro cycle. International Journal of Performance Analysis in Sport. 2014; 14: 814-828.

[32] Duhig S, Shield AJ, Opar D, Gabbett TJ, Ferguson C, Williams M. Effect of high-speed running on hamstring strain injury risk. British Journal of Sports Medicine. 2016; 50: 1536-1540.

[33] Malone S, Owen A, Mendes B, Hughes B, Collins K, Gabbett TJ. Highspeed running and sprinting as an injury risk factor in soccer: can welldeveloped physical qualities reduce the risk? Journal of Science and Medicine in Sport. 2018; 21: 257-262.

[34] Osgnach C, Poser S, Bernardini R, Rinaldo R, di Prampero PE. Energy cost and metabolic power in elite soccer: a new match analysis approach. Medicine and Science in Sports and Exercise. 2010; 42: 170 178.

[35] Tierney PJ, Young A, Clarke ND, Duncan MJ. Match play demands of 11 versus 11 professional football using Global Positioning System tracking: Variations across common playing formations. Human Movement Science. 2016; 49: 1-8.

[36] Anderson L, Orme P, Di Michele R, Close GL, Milsom J, Morgans R, et al. Quantification of Seasonal-Long Physical Load in Soccer Players with Different Starting Status from the English Premier League: Implications for Maintaining Squad Physical Fitness. International Journal of Sports Physiology and Performance. 2016; 11: 1038-1046.

[37] Malone S, Roe M, Doran DA, Gabbett TJ, Collins K. High chronic training loads and exposure to bouts of maximal velocity running reduce injury risk in elite Gaelic football. Journal of Science and Medicine in Sport. 2017; 20: 250-254.

[38] Chmura P, Konefał M, Chmura J, Kowalczuk E, Zając T, Rokita A, et al. Match outcome and running performance in different intensity ranges among elite soccer players. Biology of Sport. 2018; 35: 197-203.

[39] Buchheit M, Simpson BM. Player-Tracking Technology: Half-Full or Half-Empty Glass? International Journal of Sports Physiology and Performance. 2017; 12: S2-35-S2-41. 